\title{
In-depth analysis of road crashes involving powered two-wheelers vehicles: typical human functional failures and conditions of their production
}

\author{
Pierre Van Elslande ${ }^{\mathrm{a},{ }^{*}}$ Jean-Yves Fournier ${ }^{\mathrm{a}}$ and Magali Jaffard ${ }^{\mathrm{a}}$ \\ aIFSTTAR-MA, Chemin de la Croix Blanche, 13300 Salon-de-Provence, France
}

\begin{abstract}
Traffic safety related to motorcycle riding constitutes a growing issue all over the world. This is notably the case in France, where the important progress recently obtained toward a safer driving by car didn't prove to be so much effective for motorcycling. The present study has the purpose to give some cues on the reasons why, by characterizing the specificities of accident scenarios involving a powered two-wheeler (PTW). It is based on an in-depth accident analysis conducted by research teams composed of a psychologist and a technician intervening on the scene of the accident and later on. A sample of 384 accident cases involving a PTW is compared to a sample of 1174 car drivers involved in an accident without PTW. The results show the important differences regarding the human errors committed by PTW riders and by car drivers. They also show the interactional aspects of these different errors in the production of accidents, in the sense the physical and behavioral characteristics of PTW lead to certain errors by car drivers (perceptive failures) and these errors lead in turn to certain errors by PTW riders (prognostic failures).
\end{abstract}

Keywords: Motorcycles, Car drivers, Crashes, Human error, Interactions

\section{Introduction}

Traffic safety related to motorcycling and more generally to powered two-wheelers (PTWs) riding constitutes a growing issue all over the world, in relation with both the increasing use of this means of transport and its intrinsic vulnerability [1] as compared with other types of vehicles. This is notably the case in France, where the important progress recently obtained toward a safer driving by car didn't prove to be so much effective for motorcycling with nearly $37 \%$ of fatalities on the road while they account for $1.2 \%$ of all motor vehicle traffic in France [2].

In part, these figures result from the high level of PTWs "fragility" due to the absence of protection from the vehicle body, as the slightest collision exposes the user to injury. However, these increased rates are also due to the greater involvement of PTWs in certain types of accidents. These two characteristics attest a problem of mismatch between PTWs and the traffic system. PTWs stand out in traffic by their: specific behaviors (e.g. position on the carriageway, levels of acceleration, types of overtaking), particular dimensions, low representation in traffic, a wide range of uses and a great variety of practices and behaviors depending on the type of PTW ridden (e.g. $50 \mathrm{cc}, 125 \mathrm{cc}$ or high-powered motorcycles).

The present study had the purpose to give some cues on the specificities of the difficulties encountered by PTW riders on the road, and also the reciprocal difficulties met by the other road users when they are confronted to a PTW. This will be done by characterizing the accident scenarios involving a powered two-wheeler vehicle as compared to those not involving a PTW.

\footnotetext{
${ }^{*}$ Corresponding author. E-mail: pierre.van.elslande@ifsttar.fr
} 


\section{Method}

The research work is based on a detailed analysis of 384 traffic accidents involving PTWs, compared with a control group of accidents not involving a PTW ( $\mathrm{n}=1174)$. Accident data were collected by IFSTTAR multidisciplinary teams operating at the scene of the accidents. They cover the three components of the road system: vehicles, drivers and infrastructure. This database underwent statistical weighting in order to provide a better picture of accident causation at the French national level.

Each accident surveyed gave rise to a reconstruction in time and space of the events leading up to it. Then, a classification model of human functional failures (Van Elslande and Fouquet, 2007) was applied to the information collected by detailed interviews in order to define the breakdowns in the driver's functional chain (perception, diagnosis, prognosis, decision, execution, and overall). The contextual explanatory elements behind these human failures (endogenous or exogenous) were also defined. This allowed a cognitive analysis of the "human errors" to which the PTW riders and the other road users were subject to and of the factors of these errors. The acci- dents were analyzed from the view point of the failures encountered by the PTW riders in two conditions:

- When having an accident when alone on the road (loss of control)

- When having an accident with another road user (interactions accidents),

For this second category of accidents, were also analyzed the specific failures of the other road user involved with the PTWs.

\section{Results}

The general results show the important differences regarding the human functional failures committed by PTW riders and other road users from the control group $(\mathrm{khi} 2=56.9 ; \mathrm{p}<0.01)$. The former notably showed a more important sensitivity to diagnosis, prognosis, decision and execution failures (Table 1). These failures underlay two categories of mechanisms: one dealing with the difficulties met by PTW vis-à-vis infrastructure, the other with the difficulties they met vis-à-vis traffic.

Table 1

General distribution of functional failures for PTW $(n=384)$ and Others $(n=1174)$

\begin{tabular}{|l|l|l|l|l|l|l|l|}
\hline & Detection & Diagnosis & Prognosis & Decision & Execution & $\begin{array}{l}\text { General- } \\
\text { ized }\end{array}$ & Total \\
\hline PTW & $21.6 \%$ & $18.8 \%$ & $26.1 \%$ & $11.2 \%$ & $16.3 \%$ & $6.0 \%$ & $100 \%$ \\
\hline Others & $33.3 \%$ & $14.7 \%$ & $17.1 \%$ & $7.3 \%$ & $12.3 \%$ & $15.4 \%$ & $100 \%$ \\
\hline
\end{tabular}

\subsection{Losses of control}

Faced with infrastructure, a first problem to which PTW are more sensitive deals with a wrong diagnosis regarding a road difficulty such as a roadwork zone leading to a loss of adherence, or a bend featuring a form of "rupture" with the remainder of the route. A second general mechanism lies on a poor vehicle control when negotiating a trajectory, on a bend which, surprisingly, is often well-known by the rider. An assumption that we can propose for these 2 types of failures is that they are directly linked:

- on the one hand, to the type of vehicles that are two wheelers, and the dynamic parameters which characterize them;

- on the other hand to the type of driving that these vehicles induce, notably in terms of speed, transversal acceleration, dynamic behavior, and what can be called "sensitivity to environmental disturbances". In the sense that a difficulty which is relatively easy to manage by a car driver can become a dangerous pitfall for a PTW rider.

\subsection{Wrong interactions inside the traffic}

Faced with the traffic, PTW riders seem to be, more than others, subject to a prognostic failure, notably in the fact that they wrongly expect a regulation by the other driver, or anticipate no obstacle in limited visibility conditions. They are also more subject to decisional failures in the sense that they commit more violations of the driving safety standards in terms of speed and maneuvers which has the consequence to surprise other road users. As an explanation of these different failures is noted an overall tendency shown by riders involved in accidents to be 
overconfident in their capacities of anticipation and control.

A second category of results deals with the outstanding difference noted between the functional failures committed by car drivers when faced to a PTW or to another road user in accident cases (khi2=32.8; $<0.01$ ). As shown on Table 2 , car drivers are far more subject to a detection failure when confronted to a PTW (nearly $60 \%$ of their failures) than when they are confronted to another kind of road user $(45 \%)(\mathrm{khi} 2=16.1 ; \mathrm{p}<0.01)$. Such a result typically expresses the fragility of PTW regarding their detection by others. But the conditions under which these problems of detections are revealed are essential to analyze as they leave some space for research and for application. As a matter of fact these detection failures involve not only a lack of visual conspicuity of the PTW vehicle, but also a lack of "cognitive conspicuity" due the weak expectations of other road users regarding their potential meeting on the road and their specific behavior [4].

Indeed, this problem can be explained by the visual characteristics of PTWs, by the sensory capacities of the human perceptual system, by the atypical be- havior of PTWs and by the expectations that road users develop. The smaller dimensions of motorcycles on the carriageway are the most commonly mentioned explanatory element [5]. Cars, which are wider and have more homogenous contours and colors, are perceived more frequently by drivers in rapid information gathering. Motorbikes which are narrower and have more complex contours, provide information with high spatial frequency and are not perceived at first glance [6]. Other authors point out the importance of the cognitive aspect of the detectability problems concerning PTWs. Thus, the low expectation level that automobile drivers have concerning motorcyclists is sometimes considered as the main reason why they do not perceive them [7]. This low level of expectation is in part related to the rareness of these users in traffic [8]. It must also be pointed out that the behavior of PTWs can surprise other road users [4]. Indeed, by deviating from behavioral standards with their maneuvers, their positioning and the speeds that characterize them, PTWs confound the perceptual strategies that are usually effective for observing other automobile drivers.

Table 2

General distribution of functional failures for car drivers vs. PTW accidents $(n=218)$ and car drivers vs. others accidents $(n=905)$

\begin{tabular}{|l|l|l|l|l|l|l|l|}
\hline & Detection & Diagnosis & Prognosis & Decision & Execution & Generalized & Total \\
\hline Cars vs. PTW & $59.9 \%$ & $13.1 \%$ & $9.7 \%$ & $13.4 \%$ & $0.9 \%$ & $3.0 \%$ & $100 \%$ \\
\hline Cars vs. Others & $45.0 \%$ & $13.5 \%$ & $23.3 \%$ & $9.6 \%$ & $3.8 \%$ & $4.8 \%$ & $100 \%$ \\
\hline
\end{tabular}

\section{Discussion}

By in-depth analyzing the difficulties that both PTW riders and car drivers meet in accident situations, can be established not only the specificity that characterizes each of them but also the interactional aspect of the difficulties which confront them. Such an analysis reveals all the complexity of the mechanisms confronting PTWs and other road users. By such, it can help to defining countermeasures better suited to the complex phenomena involved.

\section{References}

[1] World Health Organization, Global status report on road safety: Time for action, Geneva, 2009.

[2] ONISR, La sécurité routière en France: bilan de l'année 2009. La Documentation Française, 2010.
[3] P Van Elslande, Classifying "human errors" in road accidents. In proceedings of the 13th Triennial Congress of the International Ergonomics Association "From experience to innovation", 1997.

[4] P Van Elslande and M. Jaffard, Typical human errors in traffic accidents involving powered two-wheelers, in Proceedings of the 27th International Congress of Applied Psychology (ICAP 2010), Ashgate, 2011.

[5] G. Wulf, P.A Hancock, and M. Rahimi, Motorcycle conspicuity: an evaluation and synthesis of influential factors, Journal of Safety Research, 20, 1989, 153-176.

[6] D. Crundall, K. Humphrey, and D. Clarke, Perception and appraisal of approaching motorcycles at junctions, Transportation Research Part F, 11, 2008, 159-167.

[7] G.J. Hole, L. Tyrrell and M. Langham, Some factors affecting motorcyclists' conspicuity. Ergonomics 39, 1996, 946-965.

[8] D.D. Clarke, P.J. Ward, C. Bartle, and W. Truman, The role of motorcyclist and other driver behavior in two types of serious accident in the UK., Accident Analysis and Prevention, 39, 2007, 974-981. 